\title{
Anne Haage: Informationsrepertoires von Menschen mit Behinderung - Barrieren und Förderfaktoren für die gleichberechtigte Teilhabe an öffentlicher Kommunikation
}

\author{
Baden-Baden: Nomos 2021. 348 Seiten. Preis: $€ 74$
}

\section{Volker Gehrau}

Angenommen: 22. Dezember 2021 / Online publiziert: 11. Januar 2022

(C) Der/die Autor(en) 2022

Rund acht Millionen Menschen in Deutschland haben einen Grad der Behinderung von mindestens 50 und gelten damit als schwerbehindert. Das bringt unterschiedliche Einschränkungen im Alltagsleben mit sich, so auch bei Zugang und Nutzung von Massenmedien. Damit ist der Personenkreis derer, die bei der Mediennutzung behindert werden, weit größer als die Reichweite vieler Medienangebote oder die Größe spezieller Zielgruppen, die oft Gegenstand kommunikationswissenschaftlicher Studien sind. Allein schon deshalb verwundert es, vorsichtig ausgedrückt, warum es bislang so wenige Studien zur Mediennutzung von Menschen mit Behinderung gibt.

Eine rühmliche Ausnahme ist die Dissertation von Anne Haage. Das Buch bietet eine gute Einführung in die Problemlage und die (wenigen) kommunikationswissenschaftlichen Studien zu diesem Thema und stellt eine von der Autorin selbst durchgeführte große Befragungsstudie über Menschen mit Behinderung und deren alltägliche Medienrepertoires vor.

Im Zentrum der Analyse steht ein Verständnis von Behinderung im Sinne der WHO als Zusammenspiel von (1) Körperfunktionen und -strukturen, (2) Aktivitäten und (3) Partizipation, das von zwei Komponenten beeinflusst wird: von Gesundheitsproblemen einerseits sowie Umwelt- und personenbezogenen Faktoren andererseits. Damit nimmt die Analyse nicht nur die klassischen Körperbehinderungen, sondern auch andere einschränkende Bedingungen in den Blick. Zudem bieten sich so unterschiedliche Anknüpfungspunkte zur alltäglichen Mediennutzung und gesellschaftlicher Teilhabe.

Auf den ersten gut 100 Seiten werden der theoretische Hintergrund und der Stand der Forschung aufgearbeitet. Das zweite Kapitel liefert eine gute Einführung in die Problematik von Behinderung und Teilhabe und deren Bezügen zur modernen Me-

Dr. Volker Gehrau $(\bowtie)$

Westfälischen Wilhelmsuniversität-Münster, Bispinghof 9-14, 48143 Münster, Deutschland

E-Mail: gehrau@uni-muenster.de 
diengesellschaft, insbesondere vor dem Hintergrund von Ungleichheit und Digital Divide. Im dritten Kapitel werden die (wenigen) Studien zur Mediennutzung von Menschen mit Behinderung in Deutschland dargestellt. Dabei liegt ein besonderer Fokus auf der MMB16-Studie des Hans-Bredow-Instituts und der TU Dortmund, an der die Autorin beteiligt war. Beide Kapitel gemeinsam bieten einen sehr guten Überblick über Arten und Verbreitung von Behinderungen in Deutschland und deren Zusammenhang mit der alltäglichen Mediennutzung sowie den rechtlichen Vorgaben dabei. Insofern lohnt sich die Lektüre nicht nur für Personen, die an der nachfolgenden empirischen Studie interessiert sind, sondern für alle, die an den Besonderheiten dieser großen Zielgruppe öffentlicher Kommunikation Interesse haben. Im vierten Kapitel wird das Konzept der Medienrepertoires eingeführt, um den Blick von Einschränkungen bei der Nutzung einzelner Medien auf das Gesamtrepertoire aller medialen Wege öffentlicher Kommunikation zu lenken. Zugleich bilden die Medien- bzw. Informationsrepertoires den theoretischen Ausgangspunkt und Rahmen der nachfolgenden empirischen Studie.

In den Kapiteln fünf bis sieben werden die Anlage und Durchführung der Befragung, das Vorgehen bei der clusteranalytischen Ermittlung der Medienrepertoires sowie eine genaue Beschreibung und Einordnung der realisierten Stichprobe dokumentiert. Personen, die bei der Lektüre solcher Studien den Fokus auf die Ergebnisse legen, wird dieser Teil vielleicht zu ausführlich erscheinen. Andererseits ist es wichtig, die besonderen Probleme bei der Konzeption und Durchführung von Befragungen von Personen mit Behinderungen vor Augen geführt zu bekommen, zumal wenn die Studie so standardisiert wie möglich durchgeführt werden soll, aber für unterschiedliche Behinderungen unterschiedliche Hilfen bei der Barrierefreiheit eingesetzt werden müssen. Bei der Einschätzung ist wichtig, dass es sich nicht um eine große, drittmittelgeförderte Studie mit Anspruch auf Repräsentativität handelt, sondern um eine von der Autorin selbst organisierte und im Rahmen von Lehrforschungsprojekten durchgeführte Studie. In Bezug auf das Sample wird eine gut begründete breite Abbildung unterschiedlicher Arten und Grade von Behinderungen realisiert, so dass die Ergebnisse vielleicht nicht im statistischen Sinne generalisierbar sind, aber trotzdem eine Übertragbarkeit auf vielfältige Alltagsphänomene erlauben. Vor allem ist die Studie methodisch interessant, weil es der Autorin hervorragend gelungen ist, ihre kommunikationswissenschaftliche Expertise mit ihrer Erfahrung in der Forschung mit und über Personen mit Behinderungen zu verbinden.

Das Herzstück der Studie bildet die im achten Kapitel vorgestellte Clusteranalyse zur Bestimmung der Informationsrepertoires. Es wurde eine optimale Clusterlösung mit sechs Gruppen identifiziert: (1) vielseitig informierte Hörbeeinträchtigte, (2) junge, social-media-orientierte Informationsbenachteiligte, (3) ältere sehbeeinträchtigte Minimalonliner*innen, (4) jüngere hochgebildete Internetnutzer*innen, (5) hochaltrige lokal interessierte digital Abseitsstehende, (6) vielseitig informierte Infotainment-Orientierte. Die Cluster werden anhand ihres Informationsrepertoires und von Persönlichkeitsmerkmalen beschrieben, verglichen und in Bezug auf ihre Möglichkeiten der gesellschaftlichen Teilhabe diskutiert. Wegen der Komplexität der Informationsrepertoires und deren Interaktion mit Behinderungen erfordert die Lektüre des Kapitels hohe Konzentration. Das liegt auch daran, dass die Autorin bei der Darstellung und Analyse der Gruppen strikt die Clusternummern benutzt. Mir 
ist die Lektüre leichter gefallen, nachdem ich mir eine Liste mit den Namen und Charakteristika der Cluster angefertigt und beim Lesen hinzugezogen habe.

Das Buch endet mit zwei kürzeren Kapiteln, in denen die Autorin die Tragweite der Behinderung von Menschen für deren Mediennutzung und gesellschaftlichen Teilhabe zusammenfasst und Möglichkeiten sowie Verpflichtungen aufzeigt, Barrieren bei der Mediennutzung und mithin Barrieren bei der gesellschaftlichen Teilhabe soweit wie möglich zu reduzieren. Da auch behinderte Menschen zur Grundgesamtheit der Mediennutzer*innen gehören, sollten sich Kommunikationswissenschaftler*innen, die sich mit Mediennutzung beschäftigen, mit der empirischen Studie befassen und überlegen, inwiefern die typischen Informationsrepertoires behinderter Menschen bei der Erhebung und Interpretation von Mediennutzungsdaten berücksichtigt werden müssen. Deshalb hoffe ich, dass die Dissertation von Anne Haage eine breite Leser*innenschaft findet.

Funding Open Access funding enabled and organized by Projekt DEAL.

Open Access Dieser Artikel wird unter der Creative Commons Namensnennung 4.0 International Lizenz veröffentlicht, welche die Nutzung, Vervielfältigung, Bearbeitung, Verbreitung und Wiedergabe in jeglichem Medium und Format erlaubt, sofern Sie den/die ursprünglichen Autor(en) und die Quelle ordnungsgemäß nennen, einen Link zur Creative Commons Lizenz beifügen und angeben, ob Änderungen vorgenommen wurden.

Die in diesem Artikel enthaltenen Bilder und sonstiges Drittmaterial unterliegen ebenfalls der genannten Creative Commons Lizenz, sofern sich aus der Abbildungslegende nichts anderes ergibt. Sofern das betreffende Material nicht unter der genannten Creative Commons Lizenz steht und die betreffende Handlung nicht nach gesetzlichen Vorschriften erlaubt ist, ist für die oben aufgeführten Weiterverwendungen des Materials die Einwilligung des jeweiligen Rechteinhabers einzuholen.

Weitere Details zur Lizenz entnehmen Sie bitte der Lizenzinformation auf http://creativecommons.org/ licenses/by/4.0/deed.de.

Dr. Volker Gehrau ist Professor für Kommunikationswissenschaft an der Westfälischen Wilhelmsuniversität-Münster. 